\title{
Review of: "Non-microbial methane emissions from tropical rainforest soils under different conditions"
}

\author{
Xueping Chen ${ }^{1}$ \\ 1 Shanghai University
}

Potential competing interests: The author(s) declared that no potential competing interests exist.

Along with biogenic methane, abiotic methane is a problem. Non-microbial methane emissions from tropical rainforest soils were studied in this manuscript under certain environmental conditions. While the findings were intriguing, there are a few points l'd like to raise:

1. While this manuscript has some intriguing findings, the majority of them just report the phenomena without providing an explanation or discussing the process behind the phenomenon/result, particularly in the discussion section. In other words, the experimental design may be deficient in demonstrating the mechanism. For example, the quantity of $\mathrm{NM}-\mathrm{CH} 4$ emitted is proportional to the amount of precursors in the soil. This research examined SOC but made no mention of its component or composition. Additionally, two forest soils with significantly different SOC contents are examined (Figure 6). Although the SOC content varies by many orders of magnitude, methane emission is exactly proportional to SOC, which seems to have nothing to do with the methane premise, but solely with the SOC content. Additionally, reactive oxygen species oxidation is considered another process for NM-CH4 production since it involves the hydroxyl radical cleaving functional groups to form $\mathrm{nm}-\mathrm{CH} 4$. The author, however, could not identify the presence of hydroxyl radicals. Additionally, although methane generation is lower in anaerobic culture than in aerobic culture, it is still significant. Studies on the influencing variables of water content discovered that a $5 \%$ water content significantly increased methane production, and the author noted in the discussion section that a suitable mechanistic explanation was lacking. As a result, I am worried about the paucity of data supporting both NM-CH4 pathways.

2. The research work are conduct by culture experiments with certain specific conditions. Is methane content monitored in situ? As in a combination of biochemical inhibitors of methanogenesis and static chambers? Is the whole process of culturing keeping sterile? For instance, is the water and gas used in the process sterilized and filtered? Additionally, the experimental location is not described well. For instance, what is the extent of a forest's coverage? Will it have an effect on the amount of sunlight/ultraviolet rays reaching the ground? How thick is litter on the soil's surface? These are the factors that have an effect on NM-CH4, and they must be discussed in detail in the M\&M. 
